\title{
The Application and Diagnostic Utility of Immunocytochemistry on Direct Smears in the Diagnosis of Pulmonary Adenocarcinoma and Squamous Cell Carcinoma
}

Michael H. Roh, M.D., Ph.D., ${ }^{1 \dagger}$ Lindsay Schmidt, M.D., ${ }^{1 \dagger}$ Jeremiah Placido, M.D., ${ }^{1}$ Sara Farmen, M.D., Ph.D., ${ }^{1}$ Kristina L. Fields, B.S., ${ }^{1}$ Anthony J. Courey, M.D., ${ }^{2}$

Douglas A. Arenberg, M.D., ${ }^{2}$ and Stewart M. Knoepp, M.D., Ph.D. ${ }^{1 *}$

The importance of subclassifying pulmonary nonsmall cell carcinoma (NSCLC) in cytologic material is becoming increasingly paramount. Occasionally, cell blocks traditionally used for ancillary studies are sparsely cellular or acellular. Hence, we investigated the diagnostic utility of immunocytochemistry for Napsin-A, TTF-1, and p63 on direct smears of NSCLC. Immunohistochemistry for Napsin-A was initially tested on a tissue microarray (TMA) composed of pulmonary adenocarcinoma. Subsequently, in 25 cases, immunocytochemistry for Napsin-A, TTF-1, and p63 was performed on cytologic direct smears. Smears were prepared from tumor cells scraped from lung resection specimens $(n=10)$, endobronchial ultrasound-guided transbronchial fine-needle aspirates $(n=13)$, and pelleted cell material from pleural effusions $(n=2)$. Immunohistochemistry utilizing the TMA revealed Napsin-A positivity in $73 \%$ of pulmonary ADCs. Next, immunocytochemistry on direct cytologic smears demonstrated a Napsin-A(+)/TTF-1(+) immunophenotype in 15 of 18 adenocarcinomas; p63 was completely negative $(n=12)$ or only focally positive $(n=3)$ in these 15 adenocarcinomas. The remaining three adenocarcinomas were negative for all three markers. All six squamous cell carcinomas were Napsin- $A(-) / T T F-1(-)$ and diffusely p63(+). In conclusion, direct smears represent a feasible and

${ }^{1}$ Department of Pathology, University of Michigan Medical School, Ann Arbor, Michigan

${ }^{2}$ Department of Internal Medicine, University of Michigan Medical School, Ann Arbor, Michigan

${ }^{\dagger}$ Michael H. Roh and Lindsay Schmidt contributed equally to this work.

*Correspondence to: Stewart M. Knoepp, M.D., Ph.D., Department of Pathology, University of Michigan Health System, 1500 E. Medical Center Drive, Ann Arbor, Michigan 48109.

E-mail: sknoepp@med.umich.edu

Received 11 January 2011; Accepted 5 February 2011

DOI $10.1002 / \mathrm{dc} .21680$

Published online 15 April 2011 in Wiley Online Library (wileyonlinelibrary.com). robust source of cellular material for immunocytochemical studies to diagnose pulmonary $A D C$ and SQC. Our method allows the cytologist to confirm on site that material for diagnostic immunocytochemistry is present thereby serving as a safeguard in instances where the cell block is of insufficient cellularity. Diagn. Cytopathol. 2012;40:949-955. @ 2011 Wiley Periodicals, Inc.

Key Words: napsin-A; TTF-1; p63; cytology; lung cancer

Lung cancer is one of the most frequently diagnosed malignancies and is the most common cause of cancer mortality in the world. ${ }^{1,2}$ Adenocarcinoma (ADC) has become the most common subtype of lung carcinoma and can be distinguished from other subtypes of nonsmall cell lung carcinoma (NSCLC), especially squamous cell carcinoma (SQC), by examining routine H\&E stained sections and slides prepared for cytologic examination. ${ }^{3}$ Nonetheless, difficulties are commonly encountered in definitively subclassifying NSCLCs, especially those that lack obvious differentiation. This is especially relevant in fine-needle aspirates (FNAs) of lung nodules and mediastinal lymph nodes. Specifically, a significant proportion of these FNAs are interpreted as NSCLC without further histologic classification, as no definitive evidence of glandular or squamous differentiation is seen.

The increasing importance of subtyping NSCLCs is underscored by recent advances in targeted chemotherapy. For instance, tyrosine kinase inhibitors and antiangiogenic compounds can be used to target pulmonary ADCs. ${ }^{4,5}$ In this context, it is important to distinguish between ADC and SQC as the use of certain drugs such as bevacizumab (Avastin) is associated with pulmonary hemorrhage in 
patients with the latter. ${ }^{6}$ Furthermore, a recent phase III study demonstrated a significant improvement in overall survival in patients with ADC treated with cisplatin/pemetrexed versus cisplatin/gemcitabine; in contrast, cisplatin/ gemcitabine was superior to cisplatin/pemetrexed for patients with SQC. ${ }^{7}$ Hence, the cytopathologist is often faced with difficult scenarios that stem from the increased demand to render a specific diagnosis based on limited material in the face of potentially deleterious consequences of NSCLC misclassification. In patients with nonlocalized, unresectable lung cancer, FNA represents a minimally invasive means to establish a tissue diagnosis. In many cases, FNA specimens represent the only opportunity to diagnose and subclassify primary lung carcinomas.

Immunohistochemistry can serve as a useful adjunct in the subclassification of NSCLCs in small biopsy specimens and FNAs. ${ }^{8,9}$ For example, immunohistochemistry using antibodies directed against TTF-1, a transcription factor that is highly specific for carcinomas of lung and thyroid origin, is frequently employed to support a diagnosis of pulmonary ADC. ${ }^{10}$ Furthermore, p63 is useful in supporting the diagnosis of SQC. ${ }^{11}$ Recently, the aspartic protease, Napsin-A, was detected by gel electrophoresis in lung $\mathrm{ADCs}^{12}$ and subsequent reports have demonstrated the utility of Napsin-A immunohistochemistry in the diagnosis of pulmonary ADCs. ${ }^{12-15}$ Specifically, Napsin-A is positive in the majority of lung ADCs and appears to be highly specific for this entity.

Currently, there are only few reports in the literature that examine the diagnostic utility of Napsin-A immunohistochemistry in cytologic samples of lung ADCs. ${ }^{16,17}$ Stoll et al. reported the sensitivity and specificity of $65 \%$ and $96 \%$, respectively, for Napsin-A immunohistochemistry for poorly differentiated pulmonary ADCs using cell block material. Cell blocks are useful for the application of immunohistochemical adjuncts when sufficient material is present in these preparations; however, additional methods are required to ensure success for cases in which the cell blocks exhibit insufficient cellularity. Given the ever increasing clinical importance of subtyping NSCLC in cytology specimens and the realization that, in some cases, cell blocks prepared from these specimens exhibit insufficient cellularity for ancillary studies, we sought to investigate the application of immunocytochemistry for Napsin-A, TTF-1, and p63 to cytologic direct smears.

\section{Methods}

The study was approved by the Institutional Review Board at University of Michigan. Immunohistochemistry for Napsin-A was initially examined using a tissue microarray (TMA) consisting of 117 lung ADCs. Next, unstained, air-dried direct smears were immunostained for 25 cases of lung NSCLCs. In 10 cases (Table I), the direct smears were prepared from scraped tumor tissue
Table I. Immunocytochemistry on Direct Smears of NSCLC in Histologically Confirmed Cases

\begin{tabular}{|c|c|c|c|c|}
\hline \multirow[b]{2}{*}{ Case } & \multirow[b]{2}{*}{ Histologic diagnosis } & \multicolumn{3}{|c|}{ Results of Immunocytochemistry ${ }^{a}$} \\
\hline & & Napsin- $A$ & $T T F-1$ & p63 \\
\hline 1 & ADC, moderately differentiated & 0 & 0 & 0 \\
\hline 2 & ADC, well differentiated & $3+$ & $1+$ & 0 \\
\hline 3 & ADC, moderately differentiated & $3+$ & $3+$ & 0 \\
\hline 4 & ADC, well differentiated & $3+$ & $3+$ & $1+$ \\
\hline 5 & ADC, poorly differentiated & $3+$ & $3+$ & 0 \\
\hline 6 & ADC, poorly differentiated & $3+$ & $3+$ & 0 \\
\hline 7 & ADC, moderately differentiated & $2+$ & $3+$ & $1+$ \\
\hline 8 & ADC, well differentiated & $1+$ & $3+$ & 0 \\
\hline 9 & SQC, moderately differentiated & 0 & 0 & $3+$ \\
\hline 10 & SQC, with treatment effect & 0 & 0 & $3+$ \\
\hline
\end{tabular}

ADC, adenocarcinoma; SQC, squamous cell carcinoma.

${ }^{\mathrm{a}} \mathrm{A}$ score of 0 denotes negative staining in all of the tumor cells. Scores of $1+, 2+$, and $3+$ denote immunoreactivity in $<10 \%, 10-50 \%$, and $>50 \%$ of the tumor cells, respectively.

present in surgically resected lung tissue associated with a histologic diagnosis of ADC or SQC. Next, unstained, air-dried direct smears were prepared from 13 consecutive endobronchial ultrasound (EBUS)-guided transbronchial FNAs initially classified as non-small cell carcinoma not otherwise specified (NSC NOS), NSC favor ADC, NSC favor SQC, ADC, and SQC based on cytomorphology. Finally, direct smears prepared using pelleted cellular material from two pleural effusion specimens obtained after centrifugation for 5 minutes at $2400 \mathrm{rpm}$ at room temperature were also used.

For the latter 15 cases (Table II), the air-dried, DiffQuik stained and alcohol-fixed, Papanicolaou stained smears were reviewed concurrently by two cytopathologists (M.H.R. and S.M.K.). For each case, a consensus diagnosis was reached and recorded prior to reviewing the immunocytochemistry slides. Specifically, a diagnosis of ADC was made when honeycomb-like sheets and threedimensional acinar clusters composed of epithelial cells exhibiting delicate, vacuolated cytoplasm and enlarged, pleomorphic, round nuclei with finely textured chromatin and conspicuous nucleoli were seen. Conversely, a diagnosis of SQC was made when there was evidence of cytoplasmic keratinization and/or large, flat, cohesive clusters of elongated cells with large nuclei exhibiting a coarse chromatin texture and prominent nucleoli. For some cases of NSCLC, an ADC or SQC was favored if there were foci suggestive of glandular or squamous differentiation, respectively. If glandular or squamous differentiation was not definitively identified, a cytomorphologic diagnosis of non-small cell carcinoma, not otherwise specified (NSC, NOS) was rendered.

Immunohistochemistry was performed on $4 \mu \mathrm{m}$ deparaffinized sections obtained from the TMA and formalinfixed, paraffin-embedded tissue blocks prepared from the 10 surgically resected lung tumors using the Ventana Autostainer (Ventana Medical System, Tucson, AZ). Unstained, air-dried direct smears were fixed in formalin for $30 \mathrm{~min}$ prior to immunostaining. Incubation with pri- 
NAPSIN-A, TTF-1, AND p63 IMMUNOCYTOCHEMISTRY

Table II. Immunocytochemistry on Direct Smears Prepared from Cytology Specimens

\begin{tabular}{|c|c|c|c|c|c|c|}
\hline \multirow[b]{2}{*}{ Case } & \multirow[b]{2}{*}{ Specimen type } & \multirow[b]{2}{*}{ Cytomorphologic diagnosis ${ }^{a}$} & \multicolumn{4}{|c|}{ Results of Immunochemistry ${ }^{a}$} \\
\hline & & & Napsin- $A$ & $T T F-1$ & p63 & Final diagnosis \\
\hline 1 & FNA & $\mathrm{ADC}$ & $2+$ & $3+$ & 0 & $\mathrm{ADC}$ \\
\hline 2 & FNA & $\mathrm{ADC}$ & $3+$ & $3+$ & 0 & $\mathrm{ADC}$ \\
\hline 3 & FNA & NSC, favor ADC & $1+$ & $2+$ & 0 & $\mathrm{ADC}$ \\
\hline 4 & FNA & $\mathrm{ADC}$ & $3+$ & $3+$ & $1+$ & $\mathrm{ADC}$ \\
\hline 5 & FNA & NSC, favor ADC & $1+$ & $2+$ & 0 & $\mathrm{ADC}$ \\
\hline 6 & FNA & $\mathrm{ADC}$ & 0 & 0 & 0 & $\mathrm{ADC}$ \\
\hline 7 & FNA & $\mathrm{ADC}$ & $1+$ & $3+$ & 0 & $\mathrm{ADC}$ \\
\hline 8 & FNA & NSC, favor ADC & 0 & 0 & 0 & NSC, favor ADC \\
\hline 9 & Pleural effusion & $\mathrm{ADC}$ & $1+$ & $3+$ & 0 & $\mathrm{ADC}$ \\
\hline 10 & FNA & SQC & 0 & 0 & $3+$ & SQC \\
\hline 11 & FNA & $\mathrm{ADC}$ & $2+$ & $3+$ & 0 & $\mathrm{ADC}$ \\
\hline 12 & FNA & NSC, favor SQC & 0 & 0 & $3+$ & SQC \\
\hline 13 & Pleural effusion & SQC & 0 & 0 & $3+$ & SQC \\
\hline 14 & FNA & NSC, NOS & 0 & 0 & $3+$ & SQC \\
\hline 15 & FNA & NSC, NOS & 0 & 0 & 0 & NSC, NOS \\
\hline
\end{tabular}

FNA, fine needle aspiration; NSC, nonsmall cell carcinoma; ADC, adenocarcinoma; SQC, squamous cell carcinoma; NOS, not otherwise specified. ${ }^{a} A$ score of 0 denotes negative staining in all of the tumor cells. Scores of $1+, 2+$, and $3+$ denote immunoreactivity in $<10 \%, 10-50 \%$, and $>50 \%$ of the tumor cells, respectively.

mary antibodies directed against Napsin-A (1:100 dilution; Novocastra, Newcastle, United Kingdom), TTF-1 (1:400 dilution; Dako, Carpenteria, CA), and p63 (1:200 dilution; Thermo Scientific, Fremont, CA) was performed for 32 min after antigen retrieval with $\mathrm{CC} 1$ buffer $(\mathrm{pH}$ $8.5)$ at $95^{\circ} \mathrm{C}$ for 20,36 , and $36 \mathrm{~min}$, respectively. Positive and negative controls were performed in parallel. Cytoplasmic staining for Napsin-A and nuclear staining for TTF-1 and p63 was scored semiquantitatively using a 3 -tier scoring system in which 0 represented to negative staining and $1+, 2+$, and $3+$ corresponded to immunoreactivity in $<10 \%, 10-50 \%$, and $>50 \%$ of the tumor cells, respectively. The immunostained slides were reviewed concurrently by two cytopathologists (M.H.R. and S.M.K.), and a consensus score for each immunostain was recorded.

\section{Results}

To verify the performance of Napsin-A immunohistochemistry, we initially immunostained a TMA consisting of 117 pulmonary ADCs. Immunoreactivity for Napsin-A was observed in $85(73 \%)$ cases. Next, immunocytochemistry for NapsinA, TTF-1, and p63 was performed on unstained, air-dried direct smears for 25 cases of NSCLC. For each case, the percentage of tumor cells that were positive for each marker was semiquantitatively assessed concurrently by two cytopathologists (M.H.R. and S.M.K.), and the consensus scores were recorded. These data are summarized in Tables I and II.

In 10 cases, the cytologic smears were prepared using surgically resected lung tumors, which provided histologic confirmation. For these cases, immunostaining was also performed in parallel on formalin-fixed, paraffin-embedded tissue sections. The histologic diagnoses for these cases were $\operatorname{ADC}(n=8)$ and SQC $(n=2)$ (Table I). Seven of the eight ADCs were positive for both Napsin-A and TTF-1; five of these tumors were negative for $\mathrm{p} 63$ (Fig. 1) whereas only focal positivity for p63 $(<5 \%$ of cells) was seen in two cases (Fig. 2). The remaining ADC was negative for all three markers. The two SQCs were diffusely p63(+) and displayed a Napsin-A(-)/TTF-1(-) immunophenotype (Fig. 3). Importantly, the immunophenotypes on immunocytochemistry were confirmed in all 10 cases as identical immunophenotypes were observed in the corresponding immunostains performed on histologic sections of tumors obtained from formalin-fixed, paraffinembedded tissue blocks.

Finally, immunohistochemistry for all three markers was performed on unstained, air-dried cytologic smears prepared from 13 EBUS-guided transbronchial FNAs and cell pellets obtained from two pleural effusions (Table II and Figs. 4, 5). A cytomorphologic diagnosis of ADC was made in seven cases; immunocytochemistry revealed positivity for both Napsin-A and TTF-1 in six of these cases. Although one of these ADCs exhibited focal p63 immunoreactivity, none of the seven ADCs exhibited diffuse p63 immunopositivity. In an additional three cases of nonsmall cell carcinoma, a diagnosis of ADC was favored; in two of these cases, demonstrating a Napsin$\mathrm{A}(+) / \mathrm{TTF}-1(+) / \mathrm{p} 63(-)$ immunophenotype assisted in rendering a final diagnosis of ADC. Next, a cytomorphologic diagnosis of SQC was made in two cases; both were negative for Napsin-A and TTF-1 while diffusely positive for p63. In an additional two cases, demonstrating a Napsin$\mathrm{A}(-) / \mathrm{TTF}-1(-) / \mathrm{p} 63(+)$ immunoprofile assisted in reporting a final diagnosis of SQC. In one case, a cytomorphologic diagnosis of a nonsmall cell carcinoma, not otherwise specified (NSC, NOS) was rendered. In this case, the tumor cells were negative for all three markers; hence, the final diagnosis remained NSC, NOS. 

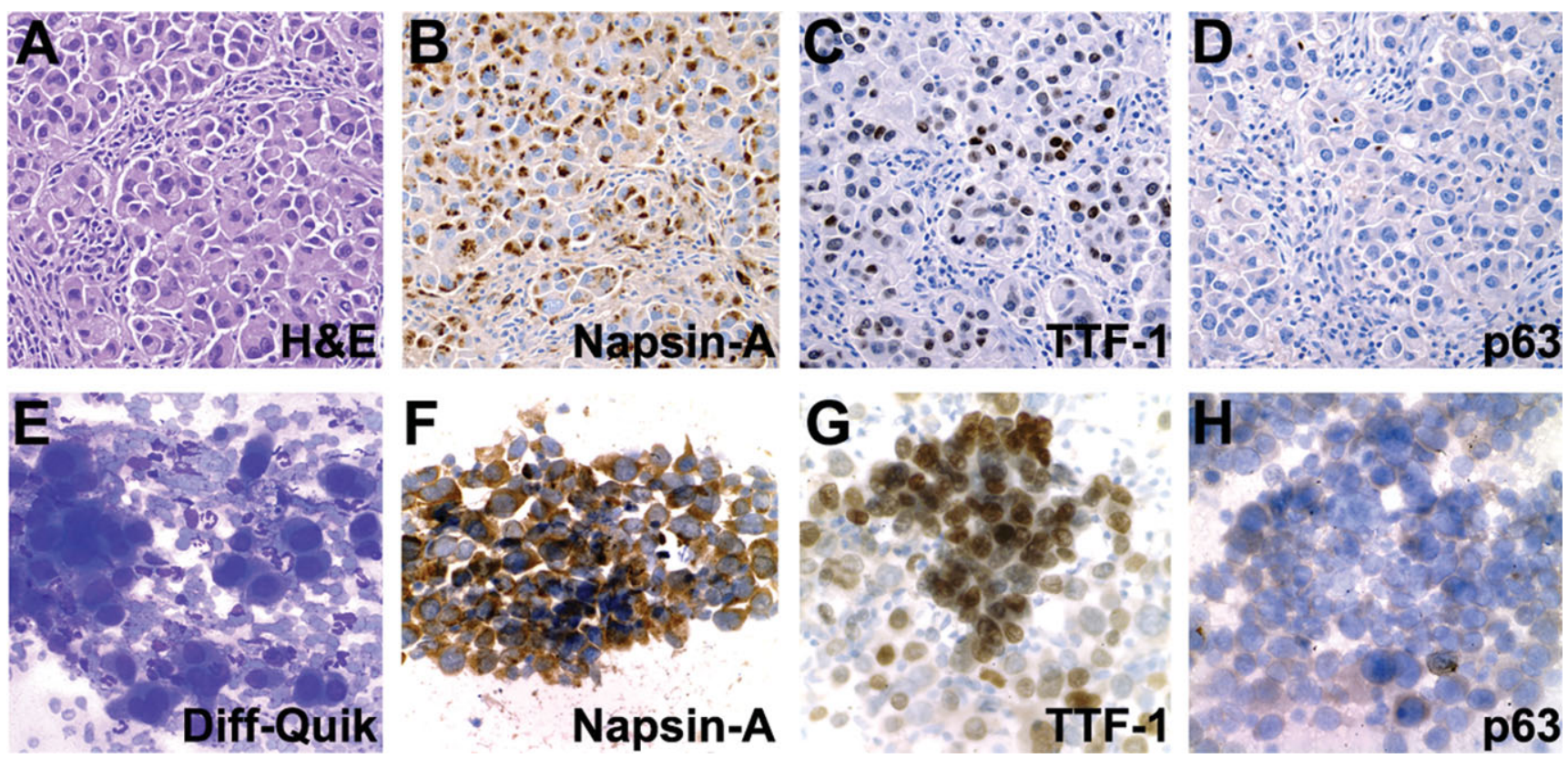

Fig. 1. Immunoperoxidase studies for Napsin-A, TTF-1, and p63 performed on histologic sections and direct smears in a case of a poorly differentiated adenocarcinoma. A-D: The upper row of photomicrographs depicts histologic sections at $\times 600$ magnification. (A) The H\&E stain depicts ill-defined solid nests composed of malignant, polygonal epithelial cells with eccentrically-placed nuclei and delicate cytoplasm. (B-D) Immunohistochemistry reveals that the tumor cells are diffusely positive for Napsin-A and TTF-1. The p63 immunostain is negative. E-H: The lower row of photomicrographs depicts cytologic direct smears at $\times 600$ magnification. (E) The Diff-Quik stained smear demonstrates a loosely cohesive to discohesive population of malignant polygonal epithelial cells. (F-H) Immunocytochemistry for Napsin-A, TTF-1, and p63 performed on direct smears also demonstrates a Napsin-A(+)/TTF-1(+)/p63(-) immunophenotype. [Color figure can be viewed in the online issue, which is available at wileyonlinelibrary.com.]
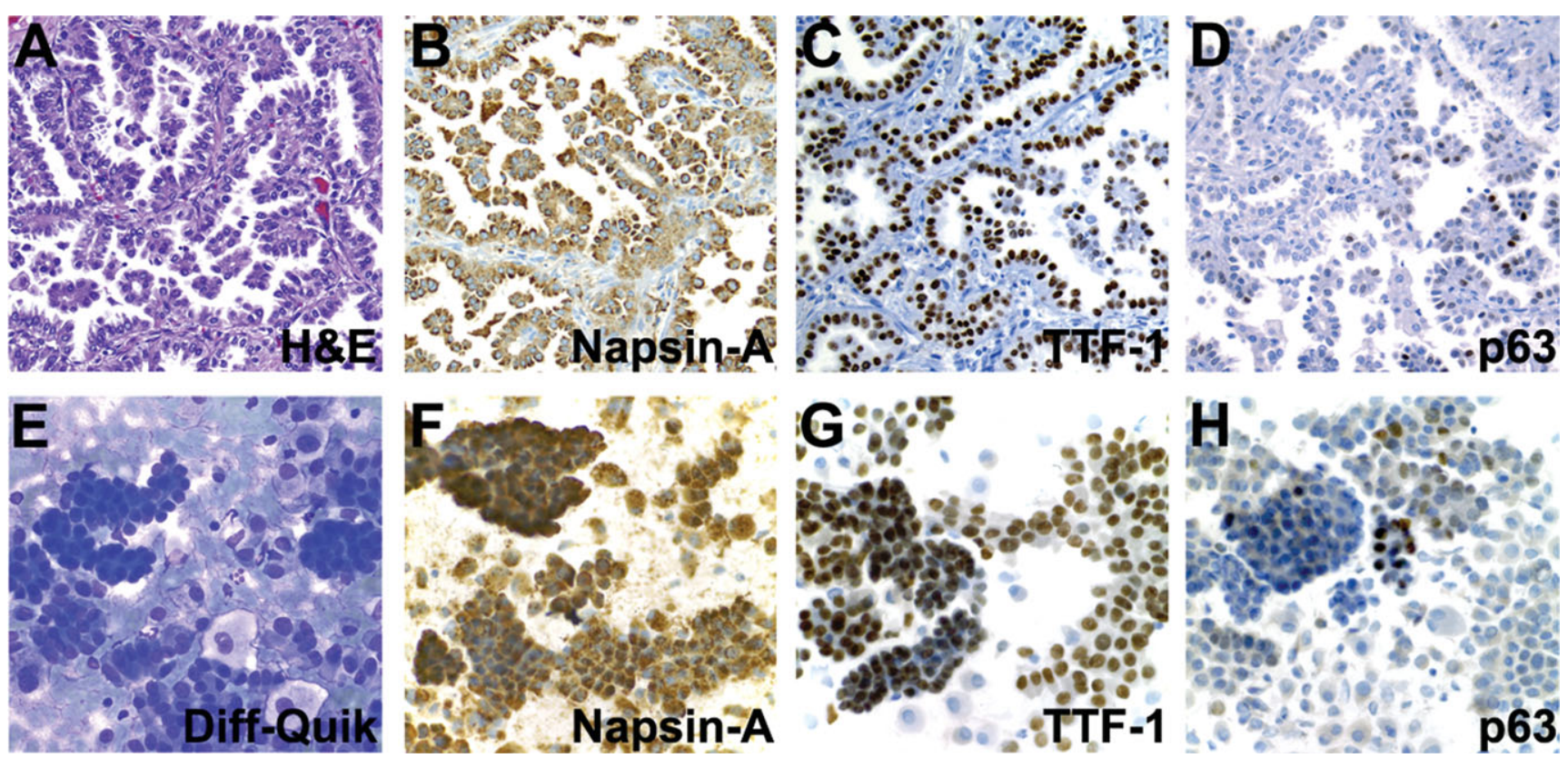

Fig. 2. Immunoperoxidase studies for Napsin-A, TTF-1, and p63 performed on histologic sections and direct smears in a case of well-differentiated adenocarcinoma. A-D: The upper four photomicrographs depict histologic sections at $\times 400$ magnification. (A) The H\&E stain depicts complex, delicate papillae composed of columnar glandular cells. (B-D) Diffuse cytoplasmic immunoreactivity for Napsin-A and diffuse nuclear positivity for TTF-1 are seen in the tumor cells. Only focal immunostaining for p63 is observed. E-H: The lower four photomicrographs depict cytologic direct smears at $\times 600$ magnification. (E) The Diff-Quik stained direct smear exhibits cohesive acinar and papillary clusters of tumor cells. (F-H) Immunocytochemistry for Napsin-A, TTF-1, and p63 performed on direct smears demonstrate the identical immunophenotype as seen on histology. [Color figure can be viewed in the online issue, which is available at wileyonlinelibrary.com.] 

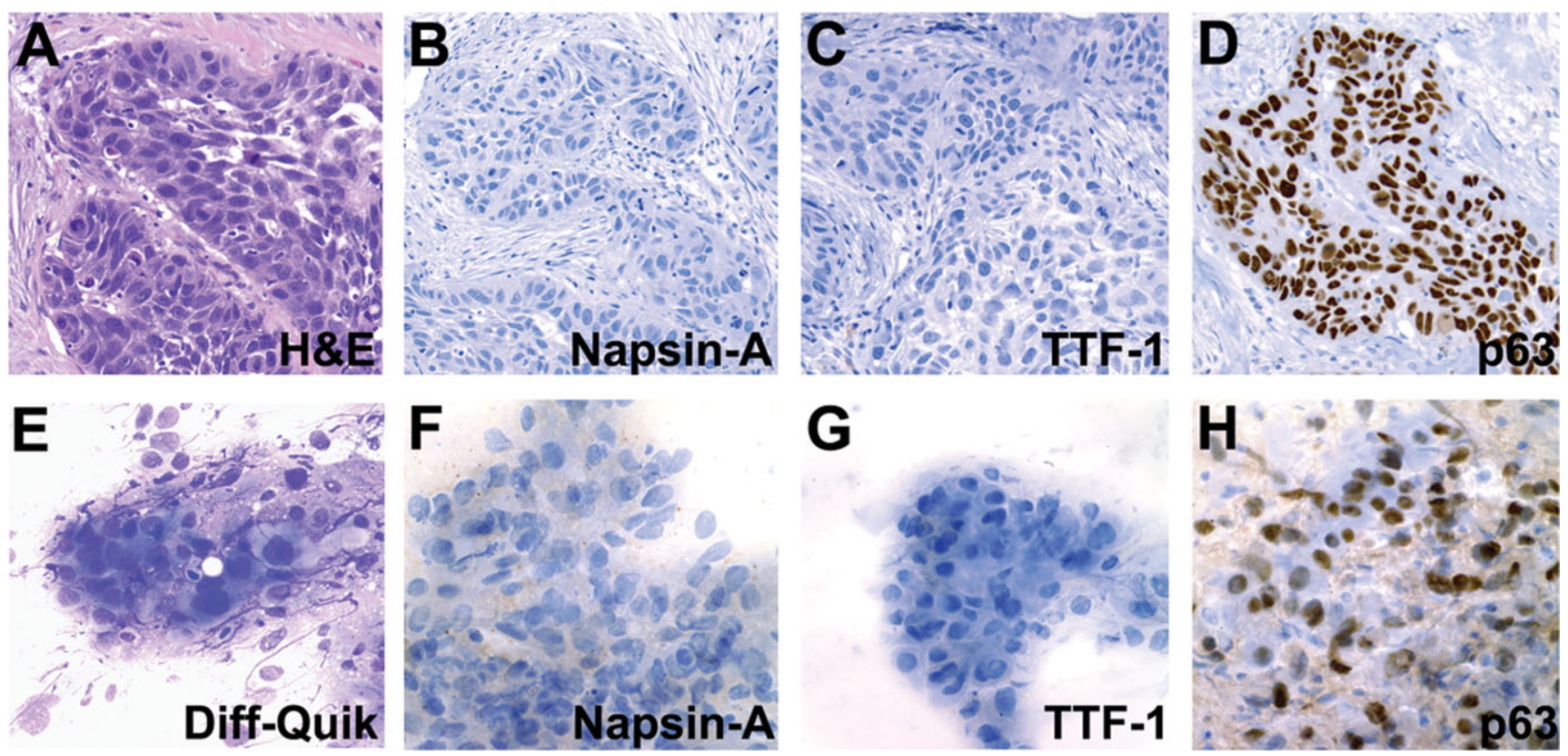

Fig. 3. Immunoperoxidase studies for Napsin-A, TTF-1, and p63 performed on histologic sections and direct smears in a case of a SQC. The upper and lower rows of photomicrographs depict histologic sections and direct smear preparations, respectively, at $\times 600$ magnification. A: An H\&E stained histologic section reveals the presence of cohesive nests of malignant squamous cells exhibiting intercellular bridging. B-D: Immunohistochemistry demonstrates that the tumor cells are diffusely immunoreactive for p63 and negative for Napsin-A and TTF-1. E: The Diff-Quik stained direct smear exhibits cohesive sheets of malignant epithelial cells with pleomorphic nuclei and dense cytoplasm consistent with squamous differentiation. $\mathbf{F}-\mathbf{H}$ : Immunocytochemistry for Napsin-A, TTF-1, and p63 performed on direct smears demonstrates the same Napsin-A(-)/TTF-1(-)/p63(+) immunophenotype. [Color figure can be viewed in the online issue, which is available at wileyonlinelibrary.com.]

\section{Discussion}

Difficulties are commonly encountered in regard to the subclassification of NSCLC, especially in small biopsies and cytologic specimens. ${ }^{18}$ In fact, the use of the NSC, NOS diagnosis has increased to over $30 \%$ of histologic samples and $37 \%$ of cytology cases of lung cancer. ${ }^{18,19}$ Nonetheless, given recent chemotherapeutic advancements in the treatment of specific subtypes of NSCLC, the need to subclassify NSCLC has never been more important. Hence, the aim of this study was to (1) ensure that material obtained at the time of fine-needle aspiration biopsy is sufficient to attempt subclassification of NSCLC; (2) confirm that diagnostic immunocytochemical stains for TTF-1, p63, and Napsin A can be performed on direct aspirate smears successfully; and (3) demonstrate that this immunocytochemical panel provides a reliable method to support a diagnosis of pulmonary SQC or ADC.

Traditionally, cell blocks have provided the mainstay for the collection of additional cellular material for ancillary immunohistochemical and molecular diagnostic studies. Despite the recognized strengths of cell blocks, such as ability to perform multiple immunostains during case workup and retain a banked archive for future studies, there are notable weaknesses. The variation in the cellularity of cell blocks, influenced by several variables including the cellularity of the lesion being targeted by
FNA, the cellularity of FNA needle rinses, effective sampling of the lesion during dedicated FNA passes for the cell block, and postprocedural handling of the needle rinse specimen, represents the foremost limitation. Furthermore, there exists a finite period of time after the FNA, during which the cell block is processed and sections are prepared for routine $H \& E$ staining, in which the cellular yield of cell block preparations are unknown. At our institution, we have observed in a series of 76 consecutive malignant cytology specimens obtained via transbronchial FNAs, $37 \%$ of cell blocks are completely acellular and an additional $20 \%$ are of minimal cellularity (unpublished observations). Prior studies examining immunohistochemical adjuncts for the diagnosis of lung cancer utilize cell block material $^{9,16,17,20}$; hence, they are inherently associated with a case selection bias whereby only cell blocks with sufficient cellularity are selected for study. The overall frequency of acellular or paucicellular cell blocks is difficult to establish. Although other laboratories would obtain differing statistics for the rate of inadequate cell blocks on FNAs, we recognized the realistic possibility that cell blocks may provide inadequate material especially in cases where the need for ancillary studies is greatest.

This realization led us to prospectively investigate whether immunocytochemistry on direct cytologic smears would permit differentiation between SQC and ADC. This method could have the advantage of being feasible 

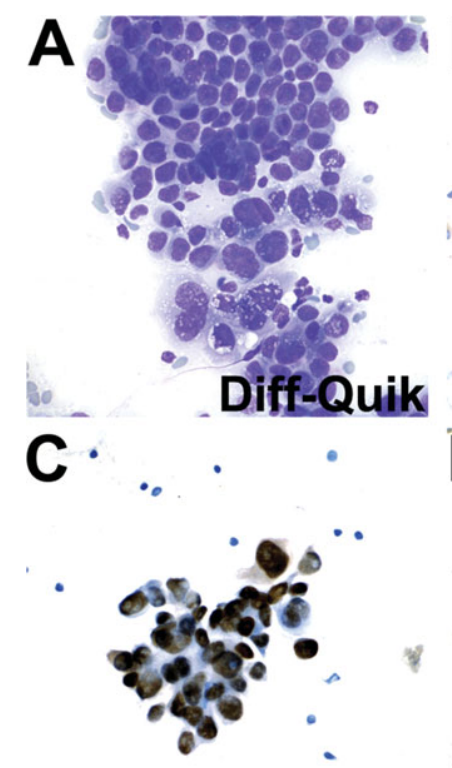

TTF-1
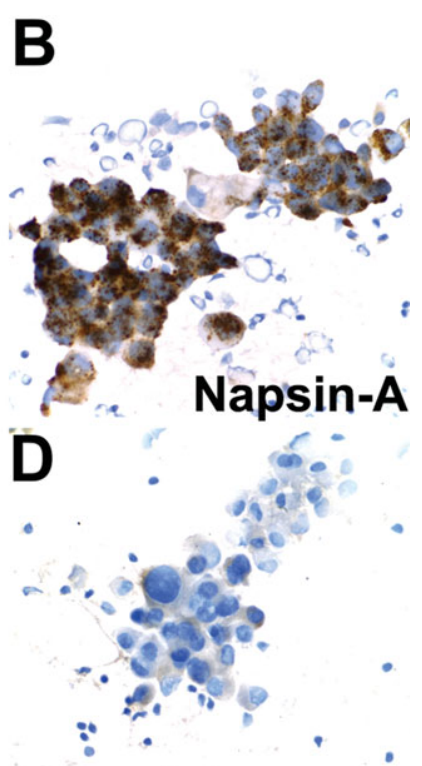

p63

Fig. 4. Immunocytochemistry for Napsin-A, TTF-1, and p63 performed on direct smears obtained from an endobronchial ultrasound-guided transbronchial fine needle aspirate of an adenocarcinoma. A: Cohesive sheets composed of tumor cells exhibiting nuclear pleomorphism, prominent nucleoli, and nuclear overlap are seen (Diff-Quik, $\times 600$ ). B-D: The tumor cells exhibit cytoplasmic immunoreactivity for Napsin-A and nuclear positivity for TTF-1; the p63 immunostain is negative $(\times 600)$. [Color figure can be viewed in the online issue, which is available at wileyonlinelibrary.com.]

for virtually every FNA procedure. Preparing multiple unstained direct smears from one or more FNA passes would allow the cytologist to confirm at the time of the procedure that adequate material for ancillary studies has been obtained. Specifically, one of the smears may be stained on-site for optimal, real-time assessment of tumor cellularity while matched slides from the same pass would be saved for ancillary immunocytochemical and/or molecular studies. Utilizing these direct smears for immunocytochemical studies could also assist in the preservation of material in the cell block for established, clinically-relevant molecular diagnostic studies as well as those that are currently being developed. Nonetheless, we have observed that, especially for FNAs in which the cell blocks exhibit insufficient cellularity, archived and freshly prepared direct smears represent a robust source of cellular material for molecular genetic studies such as EGFR and KRAS mutational analysis (Betz et al., manuscript in preparation).

In this study, diagnostic material for further workup was present in 13 consecutive EBUS-guided transbronchial FNAs cases; each FNA pass yielded on average three to four unstained smears. In addition, our method utilized formalin fixation on air-dried smears for immunocytochemistry, which has been shown to be effective in diagnostic cytology. ${ }^{21}$ This method recapitulates that used

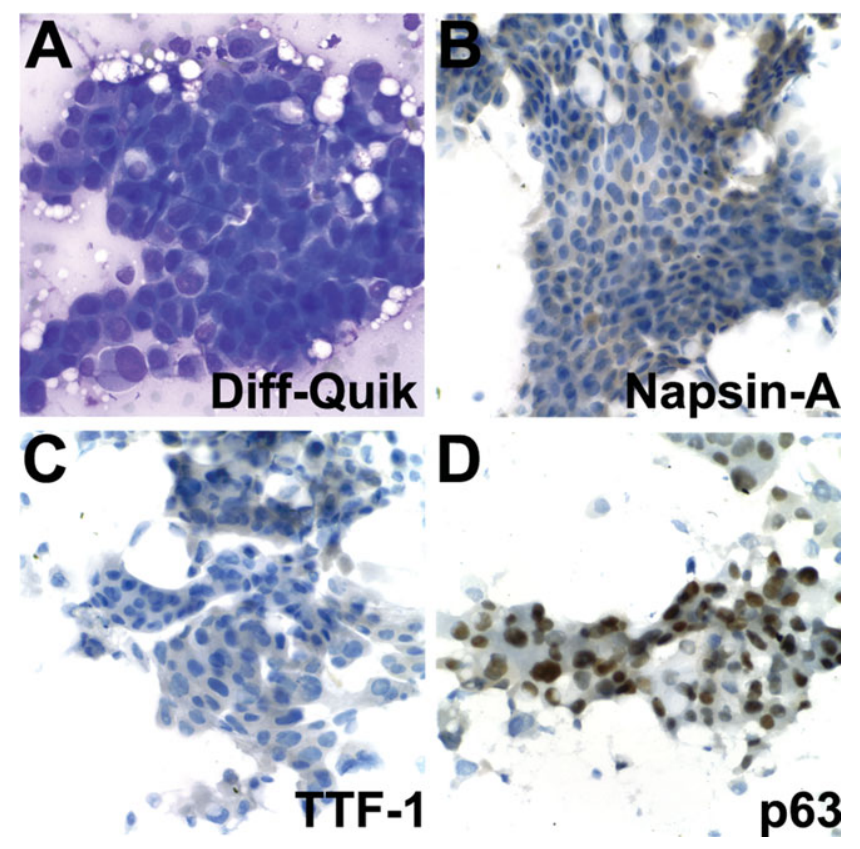

Fig. 5. Immunocytochemistry for Napsin-A, TTF-1, and p63 performed on direct smears obtained from an endobronchial ultrasound-guided transbronchial fine needle aspirate of a SQC. Cohesive, flat sheets composed of polygonal epithelial tumor cells with dense cytoplasm are appreciated in the direct smears, photographed at $\times 600$ magnification: A: Diff-Quik stained smear; B: Napsin-A immunostain; C: TTF-1 immunostain; and D: p63 immunostain. The tumor cells display a Napsin-A(-)/TTF-1(-)/ p63(+) immunophenotype, consistent with SQC. [Color figure can be viewed in the online issue, which is available at wileyonlinelibrary.com.]

on formalin-fixed, paraffin-embedded histologic sections so that similar tissue controls can be utilized. This was confirmed in our cohort of 10 cases in which unstained air-dried direct smears were obtained from surgically resected lung tumors; identical immunoprofiles for Napsin-A, TTF-1, and p63 were obtained on immunocytochemistry and immunohistochemistry.

The importance of distinguishing between ADC and SQC has been well-documented in clinical trials investigating NSCLC therapeutics. ${ }^{2,7}$ Previous studies have demonstrated that this goal can be achieved using cytologic material. ${ }^{17,20}$ Our study provides proof of an additional platform (i.e., direct smears), apart from the traditionally used cell blocks, that may be utilized to reach this goal. In our present study, TMA-based immunostaining for Napsin A of ADCs demonstrated a similar sensitivity (73\%) to that reported in previous histologic and cytologic studies. ${ }^{12,14,17,18,22}$ Furthermore, in performing immunocytochemistry on direct smears, we observed positive immunostaining for both Napsin-A and TTF-1 in 15 of 18 cases of adenocarcinomas, for a sensitivity of $83 \%$. The specificity of Napsin-A and TTF-1 positivity for adenocarcinoma was $100 \%$ in our series. For SQC, p63 expression was observed in all cases (sensitivity of $100 \%)$. In three cases of ADC, only focal staining for p63 was observed; this finding has been previously 
described and likely represents focal p63 positivity in an ADC rather than adenosquamous differentiation as there was no evidence of squamous differentiation in these cases. ${ }^{20,23}$ Furthermore, this phenomenon was also observed in our cohort of surgically resected tumors (Fig. 2). Hence, if focal (i.e., 1+) immunostaining for p63 is included, the specificity of p63 for SQC was $67 \%$. Nonetheless, the specificity for diffuse p63 immunopositivity (i.e., 3+) for SQC was $100 \%$. Our results compare favorably with the recent study by Terry et al. ${ }^{18}$ that investigated a panel of immunohistochemical markers for the subclassification of NSCLC in small biopsies. Specifically, they demonstrated a sensitivity of $59 \%$ and specificity of $94 \%$ for Napsin A and a sensitivity of $62 \%$ and specificity of $92 \%$ for TTF-1 in pulmonary ADCs. Their study also demonstrated a sensitivity of $84 \%$ and a specificity of $85 \%$ for p63 in pulmonary SQC. Overall, demonstrating immunoreactivity for Napsin-A and/ or TTF-1 along with a p63(-) immunophenotype can assist in subclassifying an NSCLC as an ADC. Conversely, a combined Napsin-A(-)/TTF-1(-) immunoprofiles associated with diffuse p63 positivity immunoprofile can assist in the diagnosis of an SQC.

In summary, our results not only confirm the utility of Napsin A, TTF-1, and p63 in the subclassification of pulmonary NSCLCs but emphasize the feasibility of direct smears as a platform for the performance of these immunostains. This can be performed on most, if not all, cytologic NSCLC specimens to ensure that appropriate and timely clinical care may be provided for patients with this diagnosis. Employing immunocytochemistry on direct smears can further cement the essential role of FNA with cytologic examination in the management of patients with NSCLC and prevent additional, potentially more invasive procedures that could result from scenarios in which insufficient material is present in the cell blocks.

\section{References}

1. Travis WD, World Health Organization, International Agency for Research on Cancer. International Association for the Study of Lung Cancer, International Academy of Pathology. 2004. Pathology and Genetics of Tumors of the Lung, Pleura, Thymus, and Heart. Lyon: IARC Press; 344 p.

2. Ladanyi M, Pao W. Lung adenocarcinoma: Guiding EGFR-targeted therapy and beyond. Mod Pathol 2008;21(Suppl 2):S16-S22.

3. Travis WD. Pathology of lung cancer. Clin Chest Med 2002;23: 65-81.

4. Besse B, Ropert S, Soria JC. Targeted therapies in lung cancer. Ann Oncol 2007;18(Suppl 9):ix135-ix142.

5. Herbst RS. Toxicities of antiangiogenic therapy in non-small-cell lung cancer. Clin Lung Cancer 2006;8(Suppl 1):S23-S30.

6. Johnson DH, Fehrenbacher L, Novotny WF, et al. Randomized phase II trial comparing bevacizumab plus carboplatin and pacli- taxel with carboplatin and paclitaxel alone in previously untreated locally advanced or metastatic non-small-cell lung cancer. J Clin Oncol 2004;22:2184-2191.

7. Scagliotti GV, Parikh P, von Pawel J, et al. Phase III study comparing cisplatin plus gemcitabine with cisplatin plus pemetrexed in chemotherapy-naive patients with advanced-stage non-small-cell lung cancer. J Clin Oncol 2008;26:3543-3551.

8. Mukhopadhyay S, Katzenstein AL. Subclassification of non-small cell lung carcinomas lacking morphologic differentiation on biopsy specimens: Utility of an immunohistochemical panel containing TTF-1, napsin A, p63, and CK5/6. Am J Surg Pathol 2011;35:1525 .

9. Rekhtman N, Brandt SM, Sigel CS, et al. Suitability of thoracic cytology for new therapeutic paradigms in non-small cell lung carcinoma: High accuracy of tumor subtyping and feasibility of EGFR and KRAS molecular testing. J Thorac Oncol 2011;6:451-458.

10. Lau SK, Luthringer DJ, Eisen RN. Thyroid transcription factor-1: A review. Appl Immunohistochem Mol Morphol 2002;10:97-102.

11. Jagirdar J. Application of immunohistochemistry to the diagnosis of primary and metastatic carcinoma to the lung. Arch Pathol Laboratory Med 2008;132:384-396.

12. Hirano T, Gong Y, Yoshida K, et al. Usefulness of TA02 (napsin A) to distinguish primary lung adenocarcinoma from metastatic lung adenocarcinoma. Lung Cancer 2003;41:155-162.

13. Chuman Y, Bergman A, Ueno T, et al. Napsin A, a member of the aspartic protease family, is abundantly expressed in normal lung and kidney tissue and is expressed in lung adenocarcinomas. FEBS Lett 1999;462:129-134.

14. Suzuki A, Shijubo N, Yamada G, et al. Napsin A is useful to distinguish primary lung adenocarcinoma from adenocarcinomas of other organs. Pathol Res Pract 2005;201:579-586.

15. Yang M, Nonaka D. A study of immunohistochemical differential expression in pulmonary and mammary carcinomas. Mod Pathol 2010;23:654-661.

16. Dejmek A, Naucler P, Smedjeback A, et al. Napsin A (TA02) is a useful alternative to thyroid transcription factor-1 (TTF-1) for the identification of pulmonary adenocarcinoma cells in pleural effusions. Diagn Cytopathol 2007;35:493-497.

17. Stoll LM, Johnson MW, Gabrielson E, et al. The utility of napsin-A in the identification of primary and metastatic lung adenocarcinoma among cytologically poorly differentiated carcinomas. Cancer Cytopathol 2010;118:441-449.

18. Terry J, Leung S, Laskin J, et al. Optimal immunohistochemical markers for distinguishing lung adenocarcinomas from squamous cell carcinomas in small tumor samples. Am J Surg Pathol 2010;34:1805-1811.

19. Ou SH, Zell JA. Carcinoma NOS is a common histologic diagnosis and is increasing in proportion among non-small cell lung cancer histologies. J Thorac Oncol 2009;4:1202-1211.

20. Khayyata S, Yun S, Pasha T, et al. Value of P63 and CK5/6 in distinguishing squamous cell carcinoma from adenocarcinoma in lung fineneedle aspiration specimens. Diagn Cytopathol 2009;37:178-183.

21. Abendroth CS, Dabbs DJ. Immunocytochemical staining of unstained versus previously stained cytologic preparations. Acta Cytol 1995;39:379-386.

22. Bishop JA, Sharma R, Illei PB. Napsin A and thyroid transcription factor-1 expression in carcinomas of the lung, breast, pancreas, colon, kidney, thyroid, and malignant mesothelioma. Hum Pathol 2010;41:20-25.

23. Au NH, Gown AM, Cheang M, et al. P63 expression in lung carcinoma: a tissue microarray study of 408 cases. Appl Immunohistochem Mol Morphol 2004;12:240-247. 